Check for updates

Cite this: J. Mater. Chem. A, 2021, 9, 21461

DOI: 10.1039/d1ta90196f

rsc.li/materials-a

\section{Retraction: Superior adsorption capacity of hierarchical iron oxide@ magnesium silicate magnetic nanorods for fast removal of organic pollutants from aqueous solution}

\author{
Shouwei Zhang, ${ }^{\text {ab }}$ Wenqing $X u^{d}$, Meiyi Zeng, ${ }^{b}$ Jie Li, ${ }^{a}$ Jiaxing $\mathrm{Li}^{\text {,a }}{ }^{\text {Jinzhang }} \mathrm{Xu}^{\mathrm{b}}$ \\ and Xiangke Wang*ac
}

Retraction of 'Superior adsorption capacity of hierarchical iron oxideamagnesium silicate magnetic nanorods for fast removal of organic pollutants from aqueous solution' by Shouwei Zhang et al., J. Mater. Chem. A, 2013, 1, 11691-11697, DOI: 10.1039/C3TA12767B.

The Royal Society of Chemistry, with the agreement of the authors, hereby wholly retracts this Journal of Materials Chemistry A article due to concerns with the reliability of the data in the published article.

The XRD spectra presented in Fig. $1 \mathrm{~A}(\mathrm{~b})$ and (c) are identical with the exception of a peak at $\sim 20$ theta in spectrum (c). Furthermore, all 3 spectra presented in Fig. 1A appear to have identical baselines in the region of 10-15 theta.

The first author also admitted that the TEM image presented in Fig. 2B was reproduced from a Materials Letter paper by Lu-Ping Zhu et al. without permission. ${ }^{1}$

The first author initially requested to retract this article, but the authors subsequently asked to correct it based on updated experimental data. The authors would like to apologise to the authors of ref. 1 and for any inconvenience to readers.

Signed: Shouwei Zhang, Wenqing Xu, Meiyi Zeng, Jie Li, Jiaxing Li, Jinzhang Xu and Xiangke Wang

Date: 11th August 2021

Retraction endorsed by Michaela Mühlberg, Executive Editor, Journal of Materials Chemistry A

\title{
References
}

1 L.-P. Zhu, G.-H. Liao, N.-C. Bing, X. Zhao and Y.-Y. Gu, Mater. Lett., 2011, 65, 1287-1290.

\footnotetext{
${ }^{a}$ Key Laboratory of Novel Thin Film Solar Cells, Institute of Plasma Physics, Chinese Academy of Sciences, P.O. Box 1126, Hefei, P. R. China. E-mail: lijx@ipp.ac.cn; xkwang@ipp. ac.cn; Fax: +86-551-65591310; Tel: +86-551-65592788

${ }^{b}$ School of Materials Science and Engineering, Hefei University of Technology, Hefei, P. R. China

${ }^{c}$ School for Radiological and Interdisciplinary Sciences, Soochow University, Suzhou 215123, P. R. China

${ }^{d}$ Department of Chemical and Environmental Engineering, Yale University, 9 Hillhouse Ave., New Haven, CT 06520, USA
} 